\title{
Operant avoidance in a rhesus monkey (Macaca mulatta) during the first month of life'
}

HERBERT KAYE AND JAMES COX BROWN UNIVERSITY

\begin{abstract}
Abstraet
Standard procedures were used to shape the barpressing avoidance response in a rhesus infant within the first month of life. By overlapping the last second of a 15-sec. tone with shock, termination of the tone was eventually sufficient stimulus change to reinforce successive approximations to the bar-press response. Eight sessions of $1 \mathrm{hr}$. or less, given over a 12-day period, yielded apparent FR 2 schedule control, the two responses being required within the first $14 \mathrm{sec}$. of tone to terminate the tone and avoid the shock. Problem
\end{abstract}

The current experiment was one of a series designed to explore the feasibility of using operant avoidance as a conditioning procedure for rhesus monkeys within the first month of life.

In a recent review of ontogeny of learning in nonhuman primates, Zimmermann \& Torrey (1965, p. 443) pointed out that no work had been done on the problem of instrumental escape and avoidance. To explain this gap, they mentioned that neonatal monkeys usually respond to aversive stimuli by "freezing." But to study developmental changes in learning it would be desirable to use a learning situation in which the same reinforcer could be employed with Ss of all ages. Such would be the case in escape and avoidance procedures wherein shock could be administered identically to all age groups. Psychophysical scaling of shock parameters would eliminate some of the problems inherent in trying to equate positive reinforcement for infants (bottle feeding) with that for adults (pellet delivery).

The runway or shuttle box would not appear to be an efficient apparatus, both because of the differences in locomotor ability of rhesus macaques below and above a month of age, and because of aifficulties in using adults in these types of test environment. The simplicity of the motor response required and the possible importance of temporal parameters indicate that a modified Skinner box would be valuable in examining developmental changes in learning.

Apparatus

A modified Skinner box, 14 in long, 13 in wide, and 18 in high, was equipped with a grid floor and a rat lever 2 in wide which protruded $3 / 4$ in from an aluminum wall. All other walls were transparent plastic, making it possible to observe the animal constantly. The box was housed in a refrigerator hull with a viewing window. Solid-state programming equipment and a VI timer presented 15-sec. tones, the last second being over- lapped with a $2.5 \mathrm{ma}$ shock delivered from an Applegate constant-current stimulator (Model 100). The tone was $1000 \mathrm{cps}$ sine-wave, generated by a Hewlett-Packard Audio Oscillator (Model 200CD). The grid floor was constructed of 3/8-in stainless-steel bars spaced about 1 in apart. Adjacent bars carried opposite polarities. $A$ red knob 1 in long and $3 / 4$ in in diameter was attached to the center of the lever during the initial shaping procedures. The cage was illuminated by a fluorescent light behind the aluminum wall panel. The timer was set for 15-sec. intertonal interval, although for the initial shaping sessions this was bypassed by a remote control switch.

\section{Procedure and Results}

The subject was a male rhesus monkey (Macaca Mulatta) born in the Brown University Primate Behavior Laboratory.

Age 18 days, session length 45 min. $S$ was placed in the box and the VI program was started. After $15 \mathrm{sec}$. a tone was presented, and 14 sec. later a 1-sec. shock was delivered. S moved over to the bar and grabbed the red knob. The next three tones were almost immediately terminated. During the next 40 trials (tonal-presentations), 34 shocks were delivered although 54 responses were made. During the remaining 60 trials, two responses were made and all shocks were received. The large number of responses early in the session and the proximity of the animal to the bar made shaping difficult during Day 1 . Ninety-four tone-shock pairings were delivered during this session. The infant was very active during no-tone intervals, but froze during tones.

Age 21 days, session length $56 \mathrm{~min}$. S remained at the far end of the cage relative to the bar. Movements made in the direction of the bar were reinforced by termination of the tone for a 15-sec. period. Movements away from the bar were punished by either turning on the tone or allowing the tone to continue through to shock. Toward the end of the session the animal began to freeze in the far corner and the session was terminated. $S$ received 50 shocks in 80 trials and responded 53 times.

Age 23 days, session length $44 \mathrm{~min}$. Shaping procedures similar to those used in the previous session were employed. After 50 trials and 15 shocks, during which 110 responses were made, shaping was discontinued and the animal was placed on CRF. During the following 54 trials, 112 responses were made and only 16 shocks were delivered. 
Age 24 days, session length $40 \mathrm{~min}$. The infant was continued on CRF, 324 responses being made in 110 trials with the delivery of only 19 shocks.

Age 25 days, session length $21 \mathrm{~min}$. CRF was continued. The red knob was removed; 24 shocks were delivered within 50 trials and 109 responses were made.

Age 28 days, session length $18 \mathrm{~min}$. CRF was maintained. The red knob was replaced, 125 responses being made in 52 trials with only 12 shocks delivered.

Age 29 days, session length $39 \mathrm{~min}$. The red knob was permanently removed, 260 responses being made in 110 trials with 18 shocks being delivered.

Age 30 days, session length $44 \mathrm{~min}$. The infant was placed on an FR 2 and responded 354 times in 110 trials, receiving 33 shocks. During the second half of the session only eight shocks were delivered. Fine-grain analysis indicated the beginning of breakrun responding, the first signs of schedule control, and possibly stimulus control.

\section{Diseussion}

The study was designed to test the feasibility of using the bar-pressing avoidance situation as a test of learning in the infant rhesus macaque within the first month of life. As such, the situation was found successful. Shaping is determined by the performance of an individual $\mathrm{S}$ and is therefore difficult to describe in detail. The procedure used with this animal was later successful with an older animal that hadbeen abandoned on a self-shaping procedure. This latter animal's avoidance response was shaped within two short sessions at about 80 days of age.

A part of the initial difficulty may have been related to the fact that in the test situation the infant was separated for the first time from the terrycloth towel on which he had been raised. The observed reaction to this is usually a self-clasping, broken sporadically by clonic spasms and jumping. The young rhesus macaque begins to walk in its home cage within the first 5 to 10 days of age and is therefore capable of exploring the test environment. Placement in the experimental cage for an hour or more without the towel during the second five days of life or earlier may help reduce some of these interfering behaviors.

Times for testing varied from 18 to $56 \mathrm{~min}$., during which at least 50 and as many as 154 trials were given. Although initial shaping took a few hours of the experimenter's time, testing the animal on CRF and higher ratios was automated. This comprises only a fraction of the time required for training and running individual infants in discrete-trial positive-reinforcement procedures.

One other relevant observation is that there was no apparent effect of the punishment procedure on food intake immediately after the session, or intake for test days compared with non-test days. Weight gain during this period was equal to that of the other infant animals in our laboratory, and within the normal range reported by Blomquist \& Harlow (1961)。

\section{Referenees}

Blomquist, A. J., \& Harlow, H. F. The infant rhesus monkey program at the University of Wisconsin Primate Laboratory. Proceedings of the Animal Care Panel, 1961, 11, 57-64.

Zimmermann, R. R., \& Torrey, C. C. Ontogeny of learning. In A. M. Schrier, H. F. Harlow, \& F. Stollnitz (Eds.), Behavior of nonhuman primates. Vol. II. New York: Academic Press, 1965. Pp. 405-447.

\section{Note}

1. This research was supported in part by Public Health Service Research Grants MH-07136 and NB-04268 to Dr. A. M. Schrier and Dr. L. P. Lipsitt, respectively. The authors thank Drs. Fred Stollnitz, Allan M. Schrier and Lewis P. Lipsitt for their critical reading of the manuscript. 